\title{
Influence of Air Pressure on the Resonance Properties of a T-Shaped Quartz Tuning Fork Coupled with Resonator Tubes
}

\author{
Mariagrazia Olivieri ${ }^{1}$, Andrea Zifarelli ${ }^{1}{ }^{\circledR}$, Giansergio Menduni ${ }^{2}$, Michele Di Gioia ${ }^{2}$, Cristoforo Marzocca ${ }^{2}$, \\ Vittorio M. N. Passaro ${ }^{2}\left(\mathbb{D}\right.$, Angelo Sampaolo ${ }^{1,3}$, Marilena Giglio ${ }^{1}\left(\mathbb{D}\right.$, Vincenzo Spagnolo ${ }^{1,3}$ (D) \\ and Pietro Patimisco $1,3, *$ \\ 1 PolySense Lab, Dipartimento Interateneo di Fisica, University and Politecnico of Bari, Via Amendola 173, \\ 70125 Bari, Italy; m.olivieri27@studenti.uniba.it (M.O.); andrea.zifarelli@uniba.it (A.Z.); \\ angelo.sampaolo@poliba.it (A.S.); marilena.giglio@poliba.it (M.G.); vincenzoluigi.spagnolo@poliba.it (V.S.) \\ 2 Department of Electrical and Information Engineering, Politecnico di Bari, 70126 Bari, Italy; \\ giansergio.menduni@poliba.it (G.M.); michele.digioia@poliba.it (M.D.G.); \\ cristoforo.marzocca@poliba.it (C.M.); vittorio.passaro@poliba.it (V.M.N.P.) \\ 3 PolySense Innovations Srl, Via Amendola 173, 70126 Bari, Italy \\ * Correspondence: pietro.patimisco@uniba.it; Tel.: +39-328-361-0558
}

\section{check for} updates

Citation: Olivieri, M.; Zifarelli, A.; Menduni, G.; Di Gioia, M.; Marzocca, C.; Passaro, V.M.N.; Sampaolo, A.; Giglio, M.; Spagnolo, V.; Patimisco, P. Influence of Air Pressure on the Resonance Properties of a T-Shaped Quartz Tuning Fork Coupled with Resonator Tubes. Appl. Sci. 2021, 11, 7974. https://doi.org/10.3390/ app11177974

Academic Editor: Detlef Kip

Received: 5 July 2021

Accepted: 25 August 2021

Published: 28 August 2021

Publisher's Note: MDPI stays neutral with regard to jurisdictional claims in published maps and institutional affiliations.

Copyright: (c) 2021 by the authors. Licensee MDPI, Basel, Switzerland. This article is an open access article distributed under the terms and conditions of the Creative Commons Attribution (CC BY) license (https:/ / creativecommons.org/licenses/by/ $4.0 /)$.

\begin{abstract}
A theoretical analysis and experimental investigation of the influence of gas pressure on resonance properties, namely, the quality factor and resonance frequency, of a T-shaped quartz tuning fork (QTF) is reported here. Two configurations are considered: a bare QTF, and a QTF coupled with a pair of resonator tubes (spectrophone). In both configurations, the effect of air on resonance frequency due to the additional inertia on prong motion and the influence of air damping on the quality factor, were analysed. By comparing the bare QTF and the spectrophone results, the effect of pressure on the acoustic coupling between the QTF and the tubes was theoretically modelled and then validated. The results show that acoustic coupling is strongly influenced by air pressure, leading to a shift of resonance frequency and a decrease in the quality factor up to $24 \%$.
\end{abstract}

Keywords: quartz tuning fork; acoustic resonator; viscous damping

\section{Introduction}

A quartz tuning fork (QTF) is an acoustic resonator widely used as a clock oscillator due to its stability and precision [1]. The ease of production and low cost of quartz crystal pushed the mass production of QTFs by using standard photolithography and chemical etching [2] or, more recently, with optical techniques consisting of direct cutting of the quartz crystal using ultrashort laser pulses [3]. This allowed the realization of different geometries and sizes, suitable for different applications [4-6]. Indeed, custom QTFs are employed in quartz-enhanced photo-acoustic spectroscopy (QEPAS) as acousto-electric transducers to detect the photo-acoustic signal generated by the absorption of modulated light from a gas sample [7]. The principle of detection in QEPAS is based on the piezoelectric properties of a quartz crystal: acoustic waves photo-generated between the prongs hit and create oscillations at one of the in-plane resonance frequencies of the QTF (in order to operate the laser source in wavelength modulation); the mechanical stress generates a strain field; electric charges appear on the surface to be collected by electrodes and converted into a voltage or a current QEPAS signal [8]. Its signal-to-noise ratio (SNR) is proportional to the squared root of the quality factor ( $Q$-factor) of the QTF resonance, defined as the ratio of the total input energy and the dissipated energy during one cycle. Furthermore, high $Q$-factors imply low dissipation losses and narrow bandwidths (typically, a few $\mathrm{Hz}$ ). These dissipation phenomena occurring while prongs are vibrating can be ascribed to: (i) air and acoustic damping, caused by interaction with surrounding air [9,10]; (ii) support losses due to the prong-support interaction [11]; and (iii) thermoelastic damping, caused 
by stress field spatial inhomogeneity leading to temperature fluctuations [12]. The main loss mechanisms depend upon the prong size and the resonance mode of the QTF. Another parameter affecting the QEPAS signal is the radiation-to-sound conversion efficiency of the gas, namely, the efficiency of the gas to convert the modulated absorbed light into sound, which is mainly determined by the non-radiative relaxation dynamics of the excited molecules [13]. Lowering the modulation frequency, i.e., the QTF resonance frequency, is essential when dealing with slow relaxing molecules (with relaxation times of few microseconds) to allow a complete release of the absorbed energy during each oscillation period. Thus, QTFs with resonance frequencies lower than $\sim 20 \mathrm{kHz}$ and a quality factor as high as possible are an interesting choice for QEPAS detection. To follow these guidelines, a new geometry with T-shaped QTF prongs was introduced in [14]. Compared with a rectangular shape, $\mathrm{T}$-shaped prongs allow a lower resonance frequency without affecting the $Q$-factor, as well as an increased stress field generated on the prong surface while the QTF is vibrating. The QTF is usually coupled with a pair of resonator tubes, allowing an enhancement of the QEPAS SNR $[15,16]$. The acoustic system composed of a QTF and a pair of resonator tubes is usually referred to as a spectrophone. In a spectrophone, an additional dissipation phenomenon can become limiting due to the interaction between the prong surface and the tubes' transversal section squeezing damping. The influences of air pressure in the resonance properties of classic shape QTFs have been reported in the literature through analytical models, and a good agreement with experiments has been demonstrated [17-19]. In [14], a new geometry of QTF was introduced that may not be modelled in a similar manner.

This work aims to analyze the influence of air pressure on the resonance properties both of a bare T-shaped QTF, and of the same QTF when acoustically coupled with two resonator tubes. A theoretical model was developed to predict the influence of air pressure both on the frequency and quality factor of the fundamental resonance mode of the QTF. The model was validated with experimental results. Furthermore, the QTF was assembled in a spectrophone with two resonator tubes. The influence of resonator tubes on the spectrophone resonance properties was investigated and compared with those of the bare QTF. The same analysis was performed on the QTF tubes at two different distances.

\section{Theoretical Model of a Quartz Tuning Fork}

\subsection{Mechanical Model}

Each prong of the QTF can be considered as a 2D free-clamped beam, since the crystal width $w$ is smaller than both the QTF thickness $T$ and length L. [20]. According to the Euler-Bernoulli beam theory, the dynamics of the vibration of a beam is described by the following differential equation [21]:

$$
E I \frac{\partial^{4} y(x, t)}{\partial^{4} x}+\rho A \frac{\partial^{2} y(x, t)}{\partial^{2} t}=0
$$

Assuming a homogeneous prong, $E$ is the Young's modulus in the QTF vibration plane, $\rho$ is the quartz density, $A$ is the prong section and $I$ is the moment of inertia. Equation (1) can be solved using the separation variable method with a free-clamped boundary condition. Thus, the resonance frequencies of the in-plane flexural modes are determined [20]:

$$
f^{\prime}{ }_{n}=\frac{1}{2 \pi} \sqrt{\frac{E I}{\rho A}} k_{n}^{2}
$$

where $k_{n}{ }^{2}=v_{n}{ }^{2} / L^{2}$ and $v_{n}$ is a number determined by the specific resonance mode $(n=0$ identifies the fundamental mode). When the QTF is vibrating in air, the prong loses energy due to interaction with the surrounding medium. This effect can be modelled in the Euler -Bernoulli theory as an additional inertia caused by the motion of the molecules in the air 
and a viscous drag force acting on the prongs. Defining $u$ as an additive mass per unit of length and $C_{d}$ as the damping parameter, Equation (1) becomes [21]:

$$
E I \frac{\partial^{4} y(x, t)}{\partial^{4} x}+(\rho A+u) \frac{\partial^{2} y(x, t)}{\partial^{2} t}+C_{d} \frac{\partial y(x, t)}{\partial t}=0
$$

In the realistic case of a negligible damping, if $u \ll \rho A$, the additive mass causes a normalized shift on the frequency of the fundamental mode given by:

$$
\Delta f=\frac{f^{\prime}{ }_{o}-f_{0}}{f_{o}^{\prime}}=\frac{u}{2 \rho A}
$$

where $f_{0}$ are the QTF resonance frequencies when prongs vibrate in air. In steady motion, the additive mass is proportional to the density of air $\rho_{\text {air }}$, thus $u=k \rho_{\text {air }}$ [22]. According to the ideal gas law, $\rho_{\text {air }}=M P / R \Theta$, where $M=28.964 \mathrm{~kg} / \mathrm{mol}$ is the molar mass, $P$ is air pressure, $R=62.3637 \mathrm{~m}^{3} \cdot$ Torr $/ \mathrm{K} \cdot \mathrm{mol}$ is the gas constant, and $\Theta$ is the QTF temperature. Air damping also affects the $Q$-factor of the QTF resonance mode. The contribution due to air damping was derived by Hosaka et al. [9]. In the viscous region, the dynamics can be described by the Navier-Stokes equation which leads to an expression for the $Q$-factor contribution $Q_{a i r}$ related to air damping given by:

$$
Q_{\text {air }}=\frac{4 \pi \rho T w^{2} f_{0}}{3 \pi \mu w+\frac{3}{4} \pi w^{2} \sqrt{4 \pi \rho_{\text {air }} \mu f_{0}}}
$$

where $\mu$ is the coefficient of the viscosity of air.

Thermoelastic and support losses are intrinsic dissipation mechanisms and can be assumed as pressure-independent. Hao et al. [11] developed a model to describe support losses as the effect of a shear force exerted from the vibrating beam on the QTF support, which excites elastic waves propagating into the support with a wavelength greater than the prong width $w$. With this assumption, the quality factor contribution $Q_{\text {sup }}$ related to support losses can be simplified as:

$$
Q_{\text {sup }}=A_{n} \frac{L^{3}}{T^{3}}
$$

with the coefficients $A_{n}$ depending on the $n$-th QTF resonance mode. Thermoelastic losses are due to the inhomogeneity of the stress field, which, in turn, causes an inhomogeneous, local increase in temperature. This temperature gradient is an additional channel of energy dissipation [12]. The corresponding Q-factor contribution $Q_{T E D}$ depends upon prong geometry and can be expressed as:

$$
Q_{T E D} \propto \frac{T^{3}}{L^{2}}
$$

These theoretical models describe each loss mechanism as standalone and their dependence on the main physical parameters is explicated. Each loss contribution is independent from the other, but all occur simultaneously for a vibrating QTF prong. The experimental investigation proposed in the next subsection aims to identify the most relevant mechanism of energy dissipation.

\subsection{Electrical Model}

The resonance properties of a QTF can be obtained by analyzing its response to a sinusoidal electrical excitation with amplitude $V_{0}$. By inverse piezoelectric effect, when voltage is applied to the prongs, a strain field and thus surface charges are generated. The electrical response of a QTF can be determined by solving its equivalent circuit $[23,24]$ represented in Figure 1. 


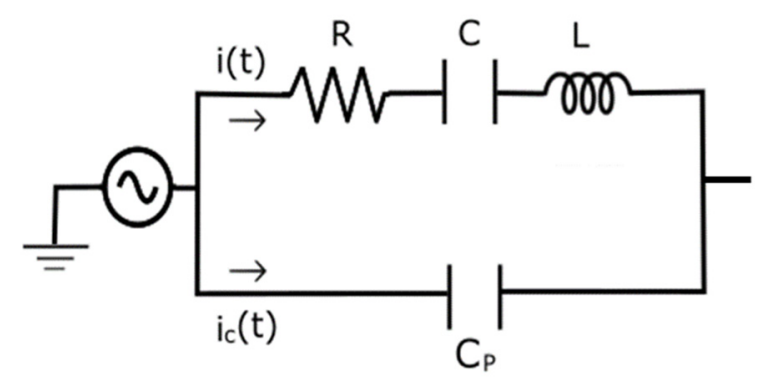

Figure 1. Equivalent circuit for a QTF. $R, C, L$ and $C_{p}$ are the electrical resistance, capacitance, inductance, and stray capacitance of the QTF, respectively.

$R, C$, and $L$ are the equivalent electrical resistance, capacitance, and inductance of the QTF, respectively. The current through the RLC circuit $i(t)$ is associated with the prong's vibration, whereas $C_{p}$ is the stray capacitance and $i_{c}(t)$ is the stray current generated by $C_{p}$. The impedance $Z$ of this circuit is given by:

$$
\frac{1}{Z(\omega)}=i \omega C_{p}+\frac{1}{i \omega L+R+\frac{1}{i \omega C}}
$$

The output current is given by the sum of $i(t)$ and $i_{c}(t)$ and is converted into a voltage signal by a transimpedance amplifier with a feedback resistor $R_{F}$. By solving the circuit represented in Figure 1, the output signal amplitude $V_{\text {out }}$ of the amplifier is retrieved as a function of the angular frequency of the excitation $\omega=2 \pi f$ :

$$
V_{\text {out }}=\frac{A \omega}{Q \omega_{0}} \frac{\sqrt{1+2 C^{\prime}\left(1-\frac{\omega^{2}}{\omega_{0}^{2}}\right)+C^{\prime 2}\left(1-\frac{\omega^{2}}{\omega_{0}^{2}}\right)^{2}+C^{\prime 2}\left(\frac{\omega}{\omega_{0} Q}\right)^{2}}}{\left(1-\frac{\omega^{2}}{\omega_{0}^{2}}\right)^{2}+\left(\frac{\omega}{\omega_{0} Q}\right)^{2}}
$$

where $A=V_{0} \cdot R_{F} / R ; Q=L \cdot \omega_{0} / R ; \omega_{0}=2 \pi f_{0}=1 / \sqrt{ } L C$; and $C^{\prime}=C_{P} / C$.

The output signal amplitude calculated by using Equation (9) is plotted in Figure $2 \mathrm{a}, \mathrm{b}$ at different $C^{\prime}$ values while keeping $A, Q$ and $f_{0}$ fixed, and (b) at different $Q$ values while keeping $A, C^{\prime}$ and $f_{0}$ fixed.

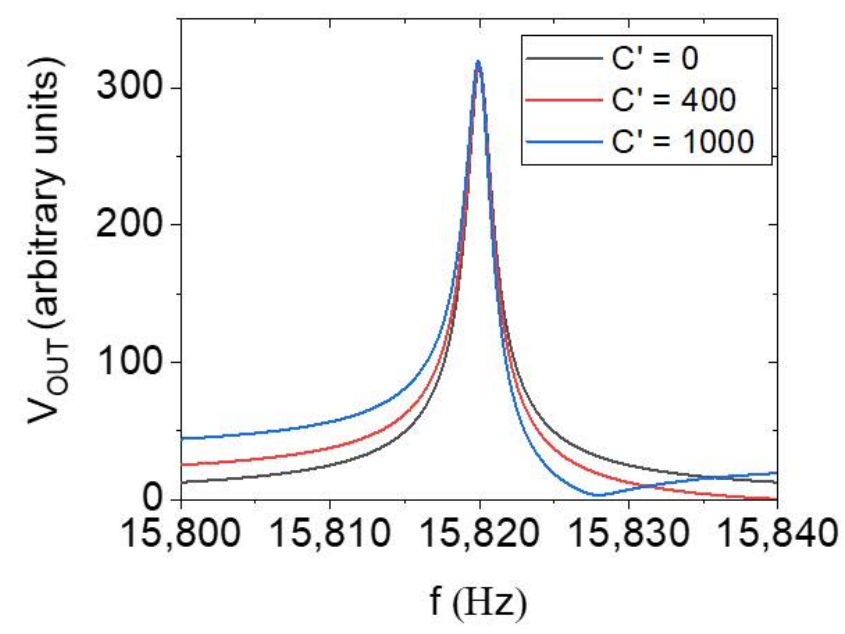

(a)

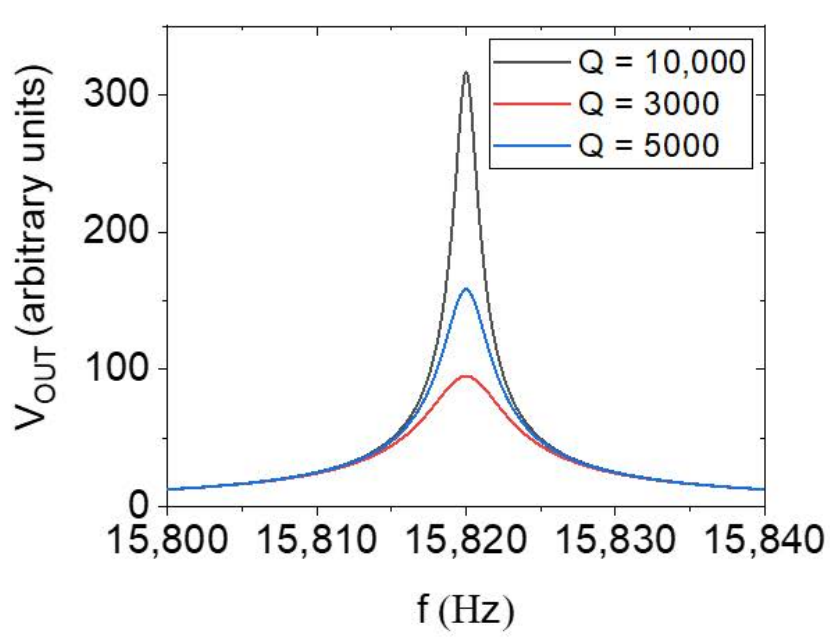

(b)

Figure 2. (a) Resonance curve of a QTF for different values of $C^{\prime}$, with $A=2 \times 10^{-8}, f_{0}=15,820 \mathrm{~Hz}, Q=10,000$. (b) Resonance curve of a QTF for different values of $Q$ with $A=2 \times 10^{-8}, f_{0}=15,820 \mathrm{~Hz}, C^{\prime}=0$. 
All curves are characterized by a peak at the resonance frequency $f_{0}$ of the QTF. As $C^{\prime}$, i.e., the stray capacitance, increases, the stray current creates a right-left asymmetry with respect to the peak value and a local minimum appears on the right side, as in the blue curve in Figure 2a. In the case in which the stray capacitance can be neglected (black curve in Figure 2a), the electrical response of the QTF can be represented by a Lorentzian line-shape. As the $Q$-factor increases (see Figure $2 b$ ) the equivalent electrical resistance of the QTF at the resonance decreases, leading to a higher resonance peak value, and the full width at half maximum (FWHM) of the resonance curve decreases.

\section{Experimental Setup}

The experimental apparatus used to electrically excite a QTF is sketched in Figure 3a. The analog output of a DAQ card was used to provide a sinusoidal electrical excitation for the QTF. The QTF was excited at its fundamental in-plane flexural mode. A LabVIEW-based software was used to vary the excitation frequency step-by-step around the resonance, with a peak-to-peak amplitude fixed to $0.5 \mathrm{mV}$. The piezoelectric current was converted into a voltage signal by a trans-impedance (TA) amplifier and acquired via the data acquisition (DAQ) card through LabVIEW-based software, which allowed retrieval of the QTF signal at the excitation frequency. The QTF was placed in stainless-steel housing (SSH) with an inlet and outlet to pump ambient air. Air passed through a first needle valve (NV1 in Figure 3a), the SSH, a second needle valve (NV2) and a vacuum pump. The valves were used to regulate the pressure inside the $\mathrm{SSH}$.

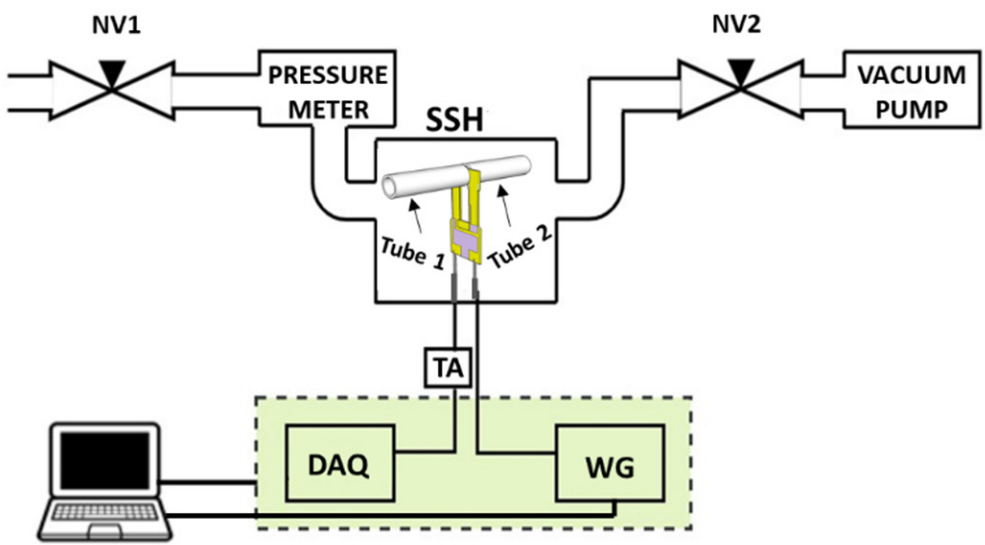

(a)

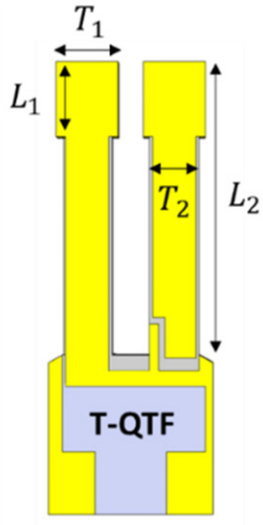

(b)

Figure 3. (a) Experimental apparatus: SSH—stainless-steel housing; NV1—needle valve 1; NV2— needle valve 2; TA—transimpedance amplifier; DAQ—data acquisition card; WFG—waveform generator. (b) Sketch of T-shaped QTF.

The pressure value was measured using a pressure display (DIGITRON 2025P). A pressure range from 10 Torr to 760 Torr was investigated. These measurements were repeated in three different configurations. Firstly, a bare T-shaped QTF was used. Then, a cylindrical V-groove (not shown in Figure 3a) was placed around the QTF to hold and fix two resonator tubes. The two tubes were mounted on both sides of the QTF (see Figure 3a) at a distance of $d=200 \mu \mathrm{m}$, perpendicular to the QTF plane with the tube center $2 \mathrm{~mm}$ below the QTF top [10]. Lastly, the distance of $200 \mu \mathrm{m}$ was halved. A sketch of the T-QTF is reported in Figure $3 \mathrm{~b}$. The geometrical parameters of the T-QTF and the tubes are summarized in Table 1. 
Table 1. Geometrical parameters of the T-QTF and of resonator tubes. $L_{1}$ and $T_{1}$ are the length and thickness of the T-shaped part of the QTF, $L_{2}$ is the total prong length, $T_{2}$ is the prong thickness, and $w$ is the crystal width. OD and ID are the outer and internal tube diameters, respectively, and $L$ is the tube length. All sizes are in millimeter units.

\begin{tabular}{cccccc}
\hline & $\boldsymbol{L}_{\mathbf{1}}$ & $\boldsymbol{L}_{\mathbf{2}}$ & $\boldsymbol{T}_{\mathbf{1}}$ & $\boldsymbol{T}_{\mathbf{2}}$ & $\boldsymbol{w}$ \\
\hline T-QTF & 2.4 & 9.4 & 2.0 & 1.4 & 0.25 \\
\hline Tubes & $\boldsymbol{L}$ & $\boldsymbol{O D}$ & $\boldsymbol{I D}$ & & \\
\hline
\end{tabular}

\section{Results}

As an example, resonance curves (black datapoints) for the bare T-QTF at two representative pressures, 10 Torr and 760 Torr, are shown in Figure 4a,b. The QTF signal is normalized and plotted as function of $1-f^{2} / f_{0}^{2}$.

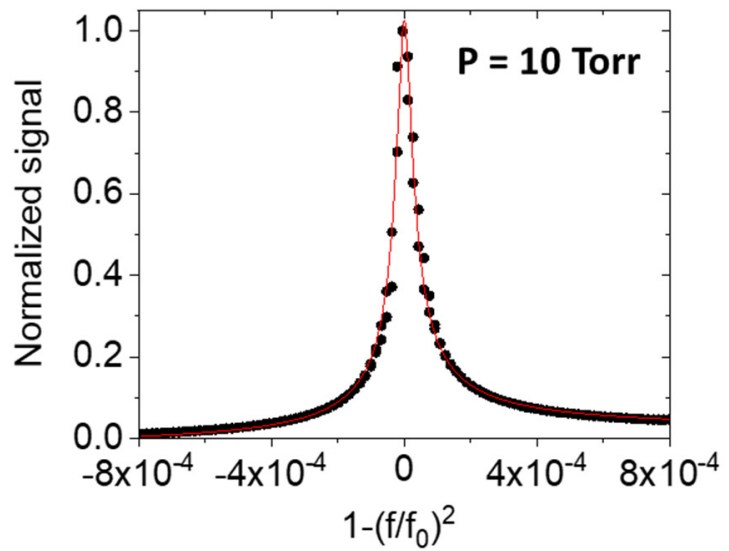

(a)

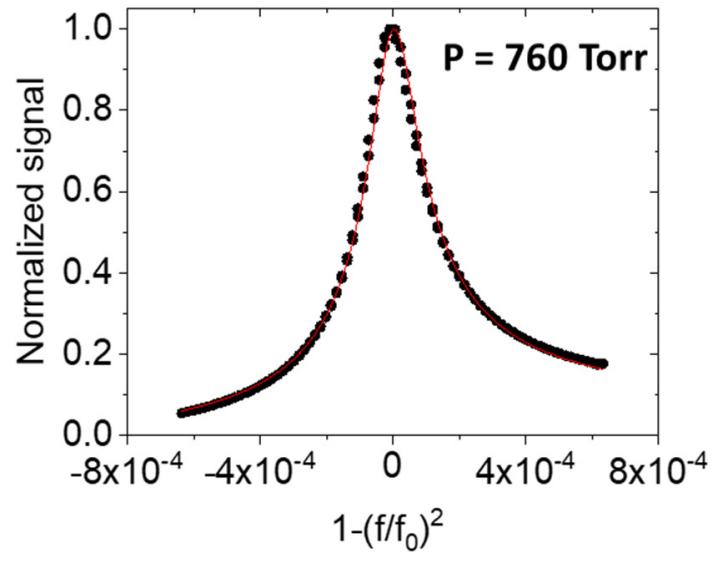

(b)

Figure 4. Resonance curve (black datapoints) for T-QTF at (a) 10 Torr, and (b) 760 Torr. The solid red line is the best fit of experimental data obtained by using Equation (10).

Taking into account the results obtained in Section 2.2, Equation (9) is used as fitting function in the form of:

$$
V_{\text {out }}=A \sqrt{\frac{(1-x) B\left(C^{\prime 2} B(1-x)+\left(C^{\prime} x+1\right)^{2}\right)}{x^{2}+(1-x) B}}
$$

where $B=1 / Q^{2}$ and $x=1-f^{2} / f_{0}^{2}$.

The $f_{0}$ value corresponding to the peak is needed for the fitting procedure. Since its value is not modified by the stray capacitance thanks to the virtual ground imposed by the transimpedance amplifier, as is observed in Figure $2 \mathrm{a}, f_{0}$ firstly can be determined by imposing a Lorentzian fit. Then, Equation (10) can be used for a second iteration fit to estimate the $Q$-factor. The best fit by using Equation (10), for the highest (760 Torr) and lowest (10 Torr) pressures at which the bare QTF was investigated, is also shown in Figure $4 \mathrm{a}, \mathrm{b}$ as solid red lines. From the preliminary Lorentzian fitting procedures, resonance frequencies $f_{0}$ are extracted and plotted as a function of the air pressure in Figure 5 (datapoints) for the three configurations. 


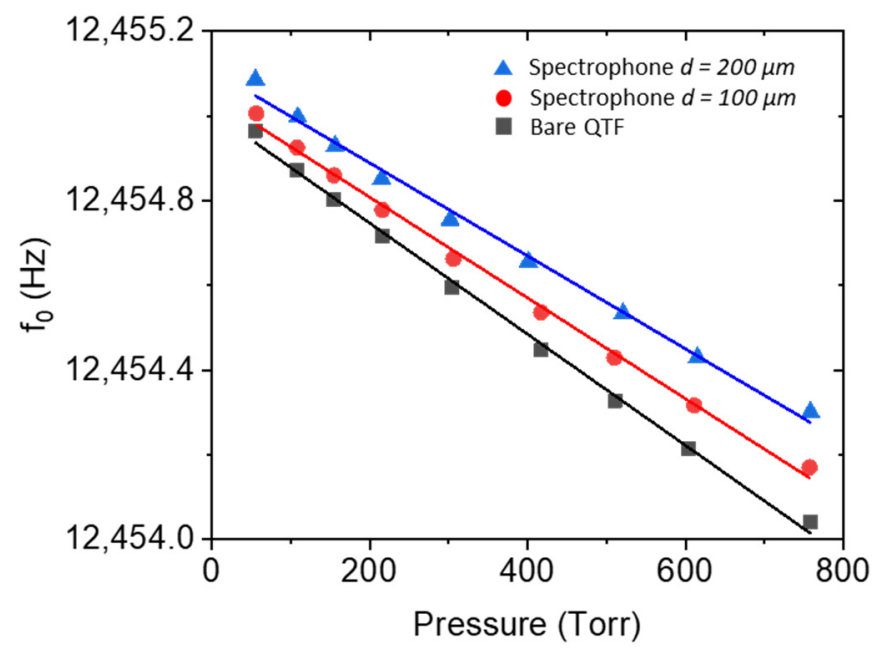

Figure 5. Resonance frequencies (datapoints) as a function of the pressure in the three configurations. The solid lines are the best linear fits.

As expected from Equation (4), $f_{0}$ decreases linearly as pressure increases. The intercept values of linear fits, i.e., the resonance frequencies in vacuum, are different for three configurations. At first glance, this seems not to be in agreement with Equation (4). It is worth noticing that the Euler-Bernoulli equation with viscous drag force term in Equation (3) is valid only when the gas is supposed to be in the viscous regime. Lowering the pressure, the gas can enter the molecular region, where damping mechanisms are caused by independent collisions of non-interacting molecules with the vibrating QTF prong. As a result, at pressures $<10$ Torr, the additive mass cannot be supposed proportional to the air density and a deviation from the linearity can also be supposed for the QTF resonance frequency. With respect to the bare QTF, the coupling with a pair of resonator tubes causes a shift of spectrophone resonance frequency. Moreover, the frequency shift increases with the pressure, although it is almost constant at pressures lower than 200 Torr. This can be explained by considering that the tubes and the QTF interact with each other through the medium in SSH at higher pressures. Thus, at low pressure, the QTF-tubes coupling is reduced and the QTF can be considered almost isolated from the tubes. For any pressure values, the frequency shift is larger when $d=100 \mu \mathrm{m}$ with respect to $d=200 \mu \mathrm{m}$. This can be explained observing that the QTF-tube distance also affects the resonator's acoustic interaction, thus leading to a greater frequency shift for a shorter QTF-tube distance. From the linear fits, the intercept value represents the fundamental vibrational mode $f^{\prime}{ }_{0}$ in vacuum whereas the slope is related to the additive mass, as described in Equation (4). Indeed, as discussed in the previous section, the additive mass per unit of length $u=u_{0}$. $P$ is proportional to the gas pressure, where $u_{0}=k M / R \Theta$ with $k$ in $\mathrm{m}^{2}$ units. $u_{0}$ represents the additive mass per unit of length and pressure, and can be retrieved from the slope $m$ of the linear fit as $m=u_{0} \cdot f_{0} /(2 \rho A)$ where $\rho=2650 \mathrm{~kg} / \mathrm{m}^{3}$ and the prong section $A=T_{2} \cdot w$. The results are listed in Table 2.

Table 2. Parameters retrieved from the linear fits shown in Figure 5. $f^{\prime}{ }_{0}$ is the intercept of the linear fit and represents the fundamental vibrational mode in vacuum; $m$ is the slope of the linear fit; $u_{0}$ is the additive mass per unit of length and pressure extracted by the slope.

\begin{tabular}{cccc}
\hline & Bare QTF & $\begin{array}{c}\text { Spectrophone } \\
\boldsymbol{d}=\mathbf{1 0 0} \boldsymbol{\mu \mathbf { m }}\end{array}$ & $\begin{array}{c}\text { Spectrophone } \\
\boldsymbol{d}=\mathbf{2 0 0} \boldsymbol{\mu \mathbf { m }}\end{array}$ \\
\hline$f_{0}(\mathrm{~Hz})$ & $12,455.01$ & $12,455.05$ & $12,455.11$ \\
\hline$m(\mathrm{~Hz} /$ Torr $)$ & $1.31 \times 10^{-3}$ & $1.19 \times 10^{-3}$ & $1.10 \times 10^{-3}$ \\
\hline$u_{0}(\mathrm{~kg} /(\mathrm{m} \times$ Torr $))$ & $1.95 \times 10^{-10}$ & $1.77 \times 10^{-10}$ & $1.64 \times 10^{-10}$ \\
\hline
\end{tabular}


The bare T-QTF shows a slightly higher value of $u_{0}$ with respect to the one obtained when it is coupled with tubes. This could be related to the fact that the presence of the tubes reduces the number of molecules surrounding the QTF which results in a decreasing of the inertia to the prong oscillation. The additional inertia of the vibrating prong due to the effect of the surrounding medium was modelled as an additive mass. Indeed, with respect to the bare QTF, the reductions in the additive mass results were $8 \%$ and $16 \%$ for QTF-tube distance $d=100 \mu \mathrm{m}$ and $d=200 \mu \mathrm{m}$, respectively.

The quality factor values are extracted by fitting the resonance curves with Equation (10). The $Q$-factor values (datapoints) are plotted as a function of the air pressure in Figure 6, for the three investigated configurations.

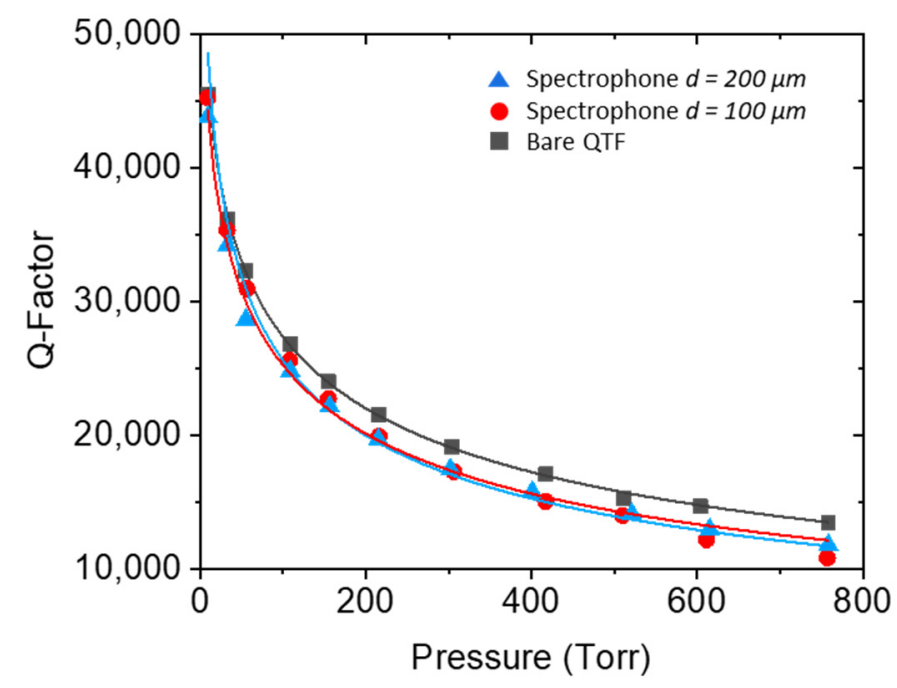

Figure 6. $Q$-factor values as a function of the air pressure (datapoints) for the three investigated configurations. Solid lines are best fits of experimental data by using Equation (12).

The trends show a strong dependence on air pressure, especially at pressures lower than 20 Torr, suggesting that the dominant loss mechanism is air damping. Moreover, working at pressures lower than 100 Torr, the $Q$-factor dramatically increases as pressure decreases, meaning that air pressure requires a higher efficient stabilization with respect to operating at pressures close to the atmospheric one. Negligible differences are observed when the QTF-tube distance is reduced from $200 \mu \mathrm{m}$ to $100 \mu \mathrm{m}$ : if the tube-QTF distance is reduced from $200 \mu \mathrm{m}$ to $100 \mu \mathrm{m}$, the spectrophone $Q$-factor is almost not affected. This "relaxes" the assembling procedure of the spectrophone, avoiding the requirement of a superfine placement of tubes. This can be explained by noting that the prong spacing $(800 \mu \mathrm{m})$ is comparable with the tube ID (see Table 1); thus, the effective interacting surface can be neglected. Indeed, while prongs are vibrating, they could lose energy via interaction with the tube walls (squeeze damping). If this additional loss mechanism is present, it should be influenced by: (i) the tube-QTF distance: the lower the distance, the higher the contribution; (ii) the air pressure: the higher the pressure, the higher the squeeze damping. Thus, in Figure 6, a squeeze damping effect should result in a deviation at higher pressures of red circles (spectrophone with $d=100 \mu \mathrm{m}$ ) towards lower values, with respect to blue triangles (spectrophone with $d=200 \mu \mathrm{m}$ ). Conversely, red circles and blue triangles should overlap at lower pressures. Instead, both datasets are quite overlapped in the whole of the investigated pressure range, demonstrating that squeeze damping is negligible. In the whole pressure range, the coupling with tubes leads to a reduction in the spectrophone $Q$-factor with respect to the bare QTF. This reduction increases as pressure increases and is almost zero at pressures lower than 100 Torr. This behavior can be explained by considering that the effect of the acoustic coupling of the high $Q$-factor QTF with the low $Q$-factor tubes, which leads to a QTF loss of energy, is reduced at low pressures, because of the reduced number of surrounding molecules. Indeed, at 10 Torr, 
the $Q$-factor of the spectrophone $(45,454)$ is comparable with the one measured for the bare QTF $(45,221)$. As a result, the surrounding air strongly affects the acoustic coupling of both resonators, namely, the tube and the QTF. It is worth noticing that datapoints in Figure 6 close to atmospheric pressure deviate from fitting with Equation (12). This is expected because Hosaka's model in Equation (5) was rewritten with the assumption that air density is proportional to pressure. This is valid only if the ideal gas law is assumed. Approaching atmospheric pressure, the ideal gas law is no longer valid, requiring a replacement with the van der Waals equation (real gas law).

As the dissipation mechanisms described in Section 2.1 are independent of each other, the overall quality factor can be written as:

$$
\frac{1}{Q(P)}=\frac{1}{Q_{0}}+\frac{1}{Q_{a i r}(P)}
$$

where $1 / Q_{0}=1 / Q_{T E D}+1 / Q_{\text {sup }}$. Using Equation (5) for $Q_{\text {air }}, Q(P)$ can be rewritten as:

$$
Q(P)=\frac{1}{C+D \sqrt{P}}
$$

where:

$$
\left\{\begin{array}{c}
C=\frac{1}{Q_{0}}+\frac{3 \mu}{4 \rho T w f_{0}} \\
D=\frac{\frac{3}{4} \sqrt{\frac{4}{4} \mu \mathrm{M}}}{4 \rho T f_{0}}
\end{array}\right.
$$

With this formulation, parameter $C$ takes into account all the pressure-independent loss mechanisms. Equation (12) was used to fit the experimental data and the results are shown as solid lines in Figure 6. The obtained fit curves were used to evaluate the difference $\Delta Q$ between the $Q$-factors of the bare QTF and the spectrophone with $d=200 \mu \mathrm{m}$, as a function of the air pressure. The result is shown in Figure 7.

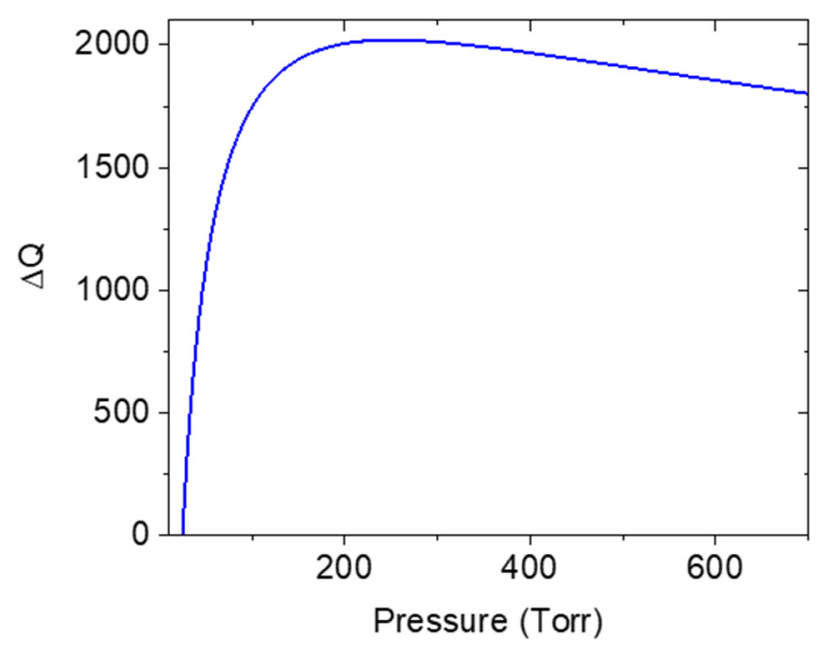

Figure 7. $\Delta Q$ as a function of the air pressure, where $\Delta Q$ is the $Q$-factor decrease when the QTF is coupled with a pair of resonator tubes at a distance of $200 \mu \mathrm{m}$.

Starting from low pressures, $\Delta Q$ quickly increases until it reaches a maximum at around 200 Torr. At $p>200$ Torr, we can assume that the influence of air on the interaction between tubes and QTF is saturated, leading to an almost flat trend with a slight decrease towards the atmospheric pressure. A reduction of $24 \%$ in the $Q$-factor of the spectrophone with respect to the bare QTF is observed at atmospheric pressure. 


\section{Conclusions}

In this work, we studied the resonance properties of a T-shaped QTF and how they are affected by acoustic coupling with a pair of resonator tubes in a spectrophone. This study was performed by analyzing the pressure-dependence of the resonance frequency and the $Q$-factor, both for the bare QTF and the spectrophone. A theoretical model was proposed and validated by experimental results. The analysis showed that dependence of the resonance properties on air pressure is well described by the proposed model in the approximation of ideal gas law. This study allowed the authors to experimentally verify how, when the QTF is vibrating in air, the effect of the surrounding medium (modelled as an additive mass for the QTF itself) affects resonance frequency, whereas energy dissipation in air reduces $Q$-factor. Moreover, additive mass is a difficult parameter to be modeled for simple geometries. This work allowed the authors to evaluate it experimentally. A reduction in the additive mass was observed for a spectrophone, when compared to the bare QTF. This means that tubes cause a shielding effect between QTF and air, leading to a reduction in the air effect on vibrating prongs. Comparing bare QTF results with those obtained with a spectrophone, it was observed that at low pressures, the quality factor of a spectrophone weakly deviates from that of the bare QTF (see Figure 6). At higher pressures, the acoustic coupling is stronger, leading to an increase in resonance frequency and a decrease in quality factor. No squeeze damping was observed when the resonator tubes were placed at a distance greater than $100 \mu \mathrm{m}$ from the QTF.

The theoretical model developed in this work can be useful for QEPAS sensing to predict working pressure, which is usually determined experimentally and can vary from gas to gas. Indeed, the QEPAS signal strongly depends on gas pressure because both the spectrophone $Q$-factor and the radiation-to-sound conversion efficiency (involving the acoustic wave generation within the gas) depends on gas pressure [6]. Although the dependence of the radiation-to-sound conversion efficiency on gas pressure has been well modelled [6], a theoretical description predicting the $Q$-factor behavior as a function of the pressure studied in this work is the fundamental step to converge for a combined theoretical model aimed at predicting the best operating pressure for QEPAS, avoiding a time-consuming experimental investigation.

Moreover, a bare QTF has recently been used as a photodetector in tunable diode laser spectroscopy (TDLAS) setup [25], showing that the photo-induced electrical signal scales linearly with the QTF accumulation time, which is proportional to its quality factor. Thus, the $Q$-factor vs. pressure trend is the necessary step to predict the enhancement factor of the photo-induced signal as a function of pressure.

Author Contributions: Conceptualization, V.S. and P.P.; methodology, G.M., A.Z., V.M.N.P. and C.M.; validation, M.O. and M.G.; formal analysis, M.O. and M.D.G.; data curation, M.O. and G.M.; writing-original draft preparation, M.O. and P.P.; writing—review and editing, A.S., M.G. and V.S.; funding acquisition, P.P. All authors have read and agreed to the published version of the manuscript.

Funding: P.P., V.S. and C.M. acknowledge funding from the European Union's Horizon 2020 research and innovation program under grant agreement No. 101016956 PASSEPARTOUT, in the context of the Photonics Public Private Partnership.

Institutional Review Board Statement: Not applicable.

Informed Consent Statement: Not applicable.

Data Availability Statement: This study did not report any data.

Conflicts of Interest: The authors declare no conflict of interest.

\section{References}

1. Friedt, J.-M.; Carry, É. Introduction to the quartz tuning fork. Am. J. Phys. 2007, 75, 415-422. [CrossRef]

2. Lee, S.; Lee, J.-Y.; Park, T.-S. Fabrication of SMD $32.768 \mathrm{kHz}$ tuning fork-type crystals: Photolithography and selective etching of an array of quartz tuning fork resonators. Mater. Corros. 2001, 52, 712-715. [CrossRef]

3. Gaudiuso, C.; Volpe, A.; Ancona, A. One-step femtosecond laser stealth dicing of quartz. Micromachines 2020, 11, 327. [CrossRef] 
4. Zheng, H.; Liu, Y.; Lin, H.; Liu, B.; Gu, X.; Li, D.; Huang, B.; Wu, Y.; Dong, L.; Zhu, W.; et al. Quartz-enhanced photoacoustic spectroscopy employing pilot line manufactured custom tuning forks. Photoacoustics 2020, 17, 100158. [CrossRef] [PubMed]

5. Duquesnoy, M. Tuning Forks in Photoacoustic Spectroscopy: Comparative Study and New Developments. Doctoral Thesis, Universitè Paris-Saclay, Gif-sur-Yvette, France, 2021.

6. Zhou, S.; Xu, L.; Zhang, L.; He, T.; Liu, N.; Liu, Y.; Yu, B.; Li, J. External cavity quantum cascade laser-based QEPAS for chlorodifluoromethane spectroscopy and sensing. Appl. Phys. B 2019, 125, 125. [CrossRef]

7. Patimisco, P.; Sampaolo, A.; Dong, L.; Tittel, F.K.; Spagnolo, V. Recent advances in quartz enhanced photoacoustic sensing. Appl. Phys. Rev. 2018, 5, 011106. [CrossRef]

8. Patimisco, P.; Sampaolo, A.; Giglio, M.; Mackowiak, V.; Rossmadl, H.; Gross, B.; Cable, A.; Tittel, F.K.; Spagnolo, V. Octupole electrode pattern for tuning forks vibrating at the first overtone mode in quartz-enhanced photoacoustic spectroscopy. Opt. Lett. 2018, 43, 1854-1857. [CrossRef] [PubMed]

9. Hosaka, H.; Itao, K.; Kuroda, S. Damping characteristics of beam-shaped micro-oscillators. Sens. Actuat. A Phys. 1995, 49, 87-95. [CrossRef]

10. Aoust, G.; Levy, R.; Bourgeteau, B.; Le Traon, O. Acoustic damping on flexural mechanical resonators. Sens. Actuat. A Phys. 2016, 238, 158-166. [CrossRef]

11. Hao, Z.; Erbil, A.; Ayazi, F. An analytical model for support loss in micromachined beam resonators with in-plane flexural vibrations. Sens. Actuat. A Phys. 2003, 109, 156-164. [CrossRef]

12. Zener, C. Internal friction in solids II. General theory of thermoelastic internal friction. Phys. Rev. 1938, 53, 90-99. [CrossRef]

13. Dello Russo, S.; Sampaolo, A.; Patimisco, P.; Menduni, G.; Giglio, M.; Hoelzl, C.; Passaro, V.M.N.; Wu, H.; Dong, L.; Spagnolo, V. Quartz-enhanced photoacoustic spectroscopy exploiting low-frequency tuning forks as a tool to measure the vibrational relaxation rate in gas species. Photoacoustics 2021, 21, 100227. [CrossRef]

14. Patimisco, P.; Sampaolo, A.; Giglio, M.; Dello Russo, S.; Mackowiak, V.; Rossmadl, H.; Cable, A.; Tittel, F.K.; Spagnolo, V. Tuning forks with optimized geometries for quartz-enhanced photoacoustic spectroscopy. Opt. Express 2019, 27, 1401-1415. [CrossRef]

15. Patimisco, P.; Sampaolo, A.; Zheng, H.; Dong, L.; Tittel, F.K.; Spagnolo, V. Quartz-enhanced photoacoustic spectrophones exploiting custom tuning forks: A review. Adv. Phys. X 2016, 2, 169-187. [CrossRef]

16. Dong, L.; Kostereve, A.A.; Thomazy, D.; Tittel, F.K. QEPAS spectrophones: Design, optimization, and performance. Appl. Phys. B 2010, 100, 627-635. [CrossRef]

17. Aoust, G.; Levy, R.; Bourgeteau, B.; Le Traon, O. Viscous damping on flexural mechanical resonators. Sens. Actuat. A Phys. 2015, 230, 126-135. [CrossRef]

18. Vignola, J.F.; Judge, J.A.; Jarzynski, J.; Zalalutdinov, M.K.; Houston, B.H.; Baldwin, J.W. Effect of viscous loss on mechanical resonators designed for mass detection. Appl. Phys. Lett. 2006, 88, 041921. [CrossRef]

19. Milde, T.; Hoppe, M.; Tatenguem, H.; Mordmuller, M.; O'Gorman, J.; Willer, U.; Schade, W.; Sacher, J. QEPAS sensor for breath analysis: A behavior of pressure. Appl. Opt. 2018, 57, 120-127. [CrossRef] [PubMed]

20. Patimisco, P.; Sampaolo, A.; Dong, L.; Giglio, M.; Scamarcio, G.; Tittel, F.K.; Spagnolo, V. Analysis of the electro-elastic properties of custom quartz tuning forks for optoacoustic gas sensing. Sens. Actuat. B Chem. 2016, 227, 539-546. [CrossRef]

21. Christen, M. Air and gas damping of quartz tuning forks. Sens. Actuators 1983, 4, 554-555. [CrossRef]

22. Sader, J.E. Frequency response of cantilever beams immersed in viscous fluids with applications to the atomic force microscope. J. Appl. Phys. 1998, 84, 64-76. [CrossRef]

23. Lee, M.; Kim, B.; An, S.; Jhe, W. Dynamic responses of electrically driven quartz tuning fork and qplus sensor: A comprehensive electromechanical model for quartz tuning fork. Sensors 2019, 19, 2686. [CrossRef] [PubMed]

24. Patimisco, P.; Scamarcio, G.; Tittel, F.K.; Spagnolo, V. Quartz-Enhanced Photoacoustic Spectroscopy: A Review. Sensors 2014, 14, 6165-6206. [CrossRef] [PubMed]

25. Dello Russo, S.; Zifarelli, A.; Patimisco, P.; Sampaolo, A.; Wei, Y.; Wu, H.; Dong, L.; Spagnolo, V. Light-induced thermo-elastic effect in quartz tuning forks exploited as a photodetector in gas absorption spectroscopy. Opt. Express 2020, 28, 19074-19084. [CrossRef] [PubMed] 